\title{
The Effect of Stitch Density and Stitching Patterns on the Puncture Resistance of High Strength Plain Woven Fabric
}

\author{
SA Suhaimi ${ }^{1 *}$, N Hassim ${ }^{1}$, MA Mohd Nor ${ }^{1}$, MR Ahmad ${ }^{2}$ and J Salleh ${ }^{2}$ \\ ${ }^{1}$ Universiti Teknologi MARA (UiTM) Negeri Sembilan Branch, Malaysia \\ ${ }^{2}$ UniversitiTeknologi MARA (UiTM), Malaysia
}

Submission: November 09, 2017; Published: February 09, 2018

*Corresponding author:SA Suhaimi, Faculty of Applied Sciences, Universiti Teknologi MARA (UiTM) Negeri Sembilan Branch, Kuala Pilah Campus, Negeri Sembilan, Malaysia, Email: suraya294@ns.uitm.edu.my

\begin{abstract}
A study was conducted on the effects of stitch density with different types of stitching patterns towards the puncture resistance of plain weave Twaron fabric. The fabrics were stitched with nylon threads using stitch densities of 4 stitches $/ \mathrm{cm}$ and 8 stitches $/ \mathrm{cm}$. Four types of stitching patterns were used namely, vertical, diagonal, square and diamond stitched patterns. A conical probe was used in the puncture resistance tests and the puncture loads required to perforate the fabric samples were recorded. The results suggest that the samples with $8 \mathrm{stitches} / \mathrm{cm}$ has higher puncture load values than the 4 stitches/cm samples, regardless of the number of layers. Among the stitching patterns, the diagonal and diamond stitched patterns showed much higher puncture load values for both the 4 stitches/cm and 8 stitches/cm samples. The study also found that three particularly different fabric failure modes during puncture resistance tests which were local yam breakages, remote yarn failure, and yam pull-out.
\end{abstract}

Keywords: Stitch density; Stitching patterns; Plain woven fabric

\section{Introduction}

Stitch density is one of the important parameters in stitching ballistic fabrics. It is described as the number of stitches/cm or stitches/inch sewn to the fabric. Stitching can improve the fabric's strength and puncture resistance against the penetration of pointed weapons or probe. Tan et al. [1] reported that stitching is highly effective in preventing fabric damage and significantly minimize the delamination of fabric. Yudhanto et al. [2] found that higher stitch density improved the tensile strength in comparison with moderate stitch density due to an effective prevention of edge-delamination of the fabric. In contrast, Karahan et al. [3] claimed that the tensile strength of plain woven Twaron T-750/ vinyl ester composites with lower stitching densities are higher than for composites with high stitching densities.

Another important parameter that contributes to the performance of fabric stitching is the stitching patterns. Ahmad et al. [4] compared four types of stitching patterns with unstitched fabric (1-inch diamond, 2-inch diamond, perimeter and diagonal stitching patterns). The study showed that all the stitched fabrics have better ballistic impact resistance than the unstitched fabric. The results from the study discovered that the 2-inch diamond stitched fabrics have the highest ballistic impact resistance, followed by the perimeter, and diagonal stitched patterns. A study on stitched self-reinforced polypropylene composites by Nanthagopal et al. [5] found that the $+45^{\circ} /-45^{\circ}$ direction gave better flexural properties and impact resistance in comparison with other stitching directions due to assembly of warp and weft threads in both directions. In another study, Suhaimi et al. [6] investigated on the effects of different stitching patterns towards the puncture resistance of multiple layers of stitched fabric. It was reported that the 4-layer diamond stitched fabric gave the highest puncture load values. The additional number of fabric layers contributed to the increment of puncture resistance to withstand the high load of penetration. The diamond stitched patterns have better movement of fabric control and hence higher puncture resistance properties than other stitching patterns. Carr et al. [7] found that the amount of energy absorbed during ballistic impacts for diamond quilted was $14 \%$ and $22 \%$ higher compared to non-quilted and square quilted depending on the number of fabric layers.

Studies by Karahan et al. [8] concluded that as stitch density increased, the tensile strength and strain decreased because the 
stitching points represented the weak zones of the samples. The study also found significant differences between the longitudinal and diagonal strength values for unstitched and stitched samples. In another study, Karahan et al. [3] also found that the tensile strength in the longitudinal direction increased significantly for lower stitch densities, did not change significantly for medium stitch densities, and decreased significantly for higher stitch densities. In the diagonal direction, there were increment of strength for lower stitch densities and decrement of strength for middle and high stitch densities. The importance of stitching parameters were also supported by Wang et al. [9] who studied on the quasi-static puncture resistance behaviours of highstrength polyester fabric for soft body armour. The structure, weft density and the number of fabric layers affected the quasistatic puncture resistance property of the fabrics. The contact pressure and friction of the probe against fibres became the main hindrance during the quasi-static puncture process and the breakage of the fibres during the penetration was caused by the bend and tensile deformation.

This paper investigates the effect of stitch density of the puncture resistance properties of high strength fabric system.
Fabrics were stitched with nylon threads using two types of stitch density, which were 4 stitches/cm and 8 stitches/cm to see the performance on four types of stitching patterns; vertical, diagonal, square and diamond stitched pattern.

\section{Methodology}

\section{Materials}

Plain weave Twaron CT 716 was used as the fabric material. The areal density of the fabric was $280 \mathrm{~g} / \mathrm{m}^{2}$ with $1000 \mathrm{dTex}$ yarn count. The square fabric has a fabric density of $12 \mathrm{ends} / \mathrm{cm}$ and 12 picks $/ \mathrm{cm}$. A nylon thread with 573 dTex linear density was used as the sewing thread for stitching the fabric. Samples of 2-layer, 3-layer and 4-layer Twaron fabrics were used as the testing parameters.

\section{Experiments}

Two types of stitch density were used, which were 4 stitches/cm and 8 stitches/cm as shown in (Figure 1). Four types of stitching patterns were used in the study: vertical, diagonal, square and diamond stitched patterns as shown in (Figure 2).
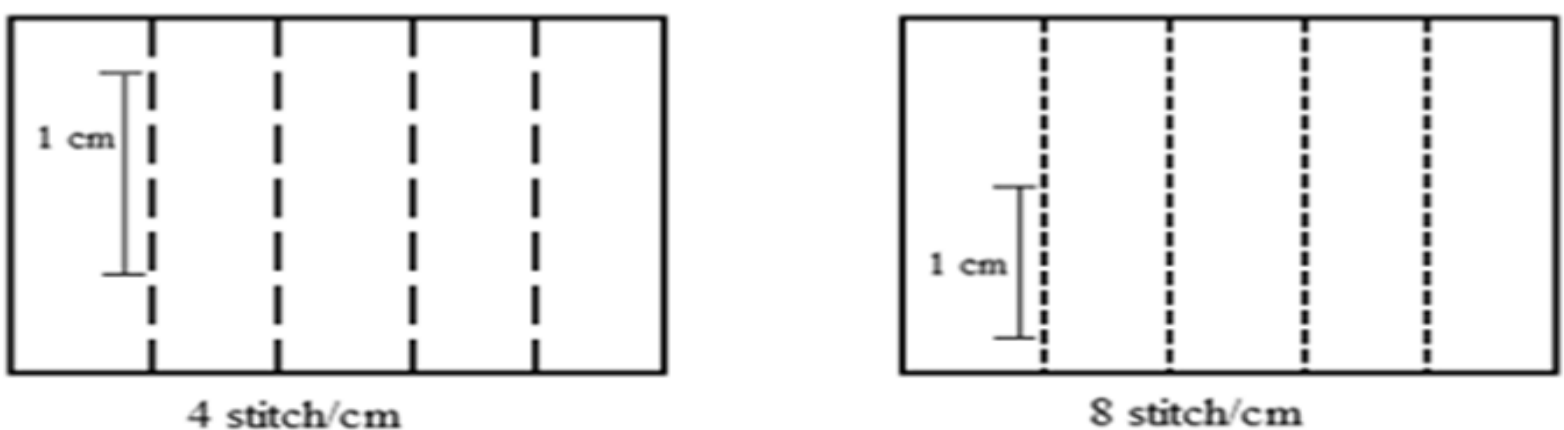

Figure 1: Types of Stitch Density.
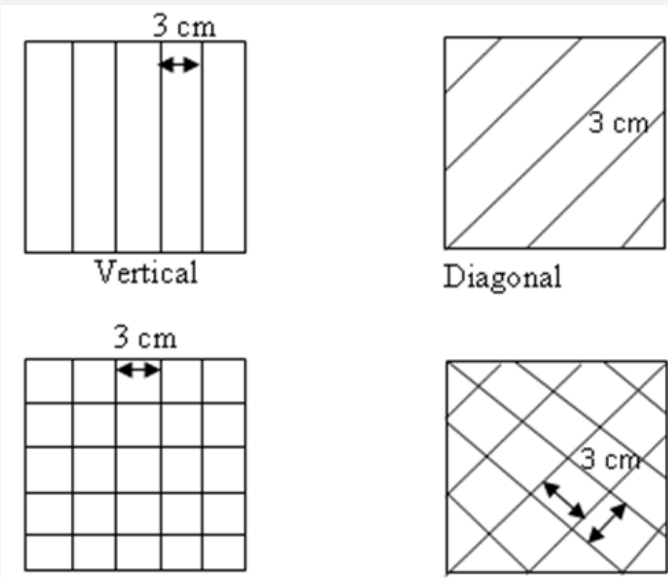

Square

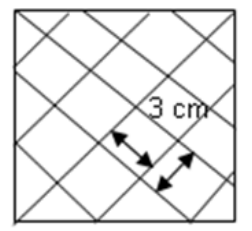

Diamond

Figure 2: Types of Stitch Pattern. 
A conical shape of puncture probe was used in the puncture resistance test. A circular jig was used to clamp the fabric samples and placed on the SDL Testometric Testing machine. The puncture probe was attached with a load cell at the upper clamp of the test machine as shown in (Figure 3). The puncture probe was made to move down at a speed of $100 \mathrm{~mm} / \mathrm{min}$ until sample penetration and rupture occurred. The average maximum load achieved in the test was calculated and reported.

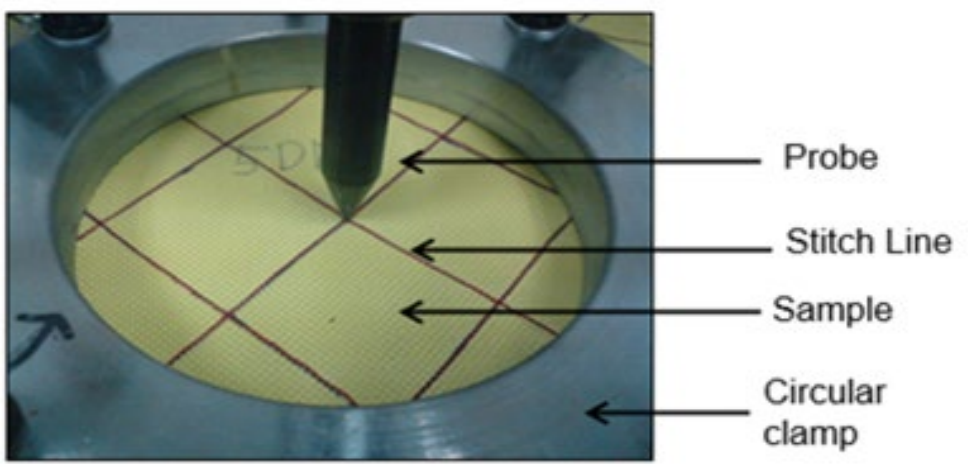

Figure 3: Position of Conical Puncture Probe during Puncture Resistance Test.

\section{Results and Discussion}

\section{Effect of stitch density}

The effect of stitch density was studied to determine which type of stitch density has the higher value by comparing the results between the 4 stitches/cm and 8 stitches/cm samples as shown in (Figure 4-6).

For the 2-layer fabric, the puncture load of all samples with 8stitches/cm was higher than 4 stitches/cm for all type of stitch patterns as shown in (Figure 1). The diagonal stitched pattern gave the highest difference which was about $40.7 \%$.

From Figure 5, for 3-layer result, the puncture load of 8 stitches/cm samples was higher on vertical and diagonal stitched patterns, but the puncture load was slightly lower on square and diamond stitched patterns. Again, the diagonal stitched pattern showed highest difference with $11.5 \%$ higher than 4 stitches/ cm samples.
For 4-layer result based on Figure 6, the square stitched pattern showed highest difference of puncture load between both stitch densities which is around $5 \%$. The puncture load for 8 stitches/cm samples was higher than 4 stitches/cm samples on all type of stitch patterns.

Briefly, the samples with 8 stitches/cm value has higher puncture load value than 4 stitches/cm samples from 2-layer to 4-layer. It can be anticipated that fabrics that were stitched with high stitch density (8stitches/cm) contributed to the higher puncture load due to the stitching distributed the energy and absorbed the penetration load from the puncture probe throughout the fabric system. The increment of fabric layer with higher stitch density could hold the multi-layer fabric better compared with the fabric that was stitched with lower stitch density.

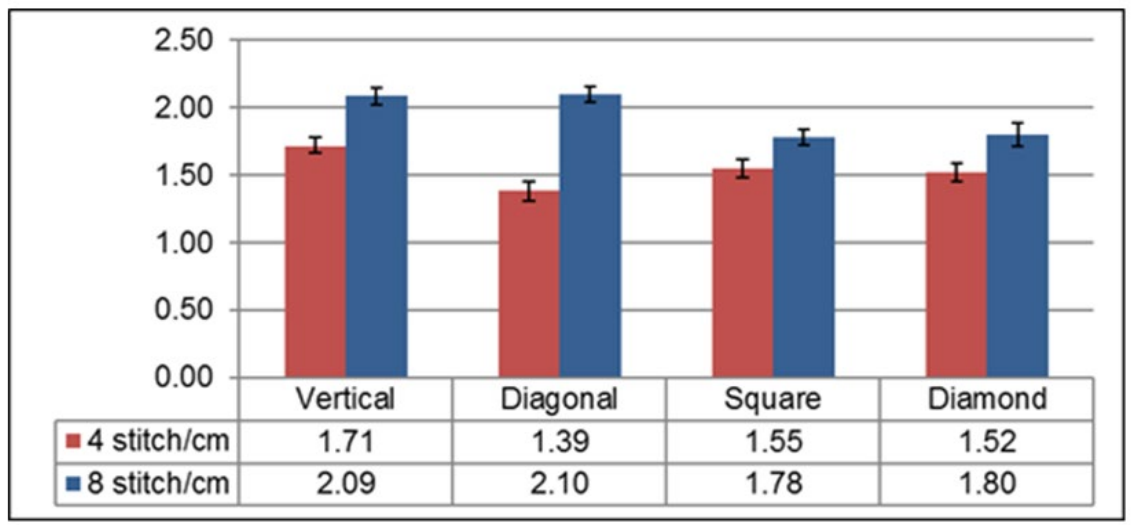

Figure 4: Puncture Load for 2-Layer (kN). 


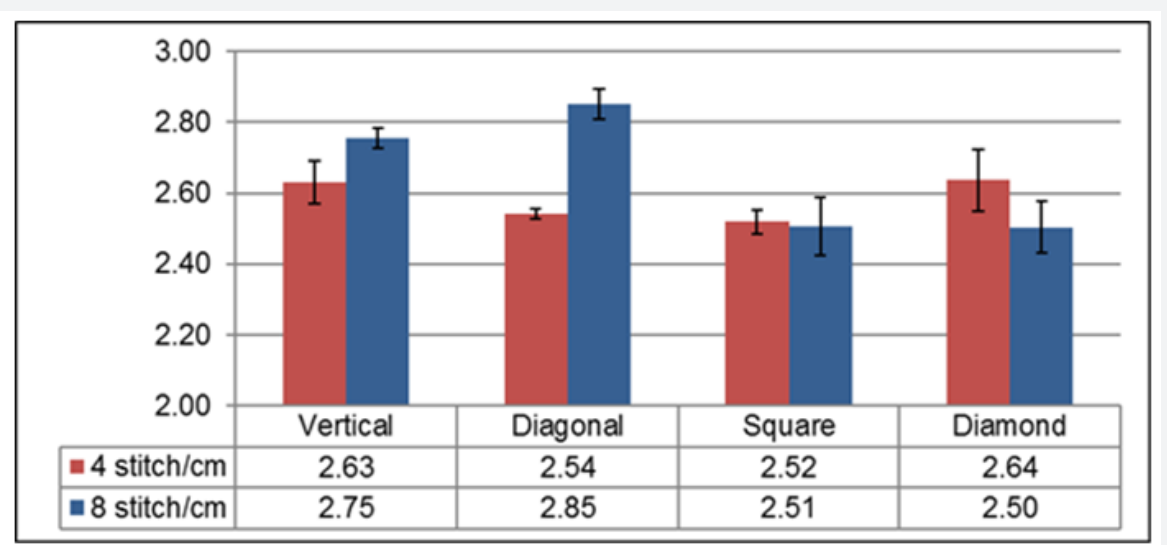

Figure 5: Puncture Load for 3-Layer (kN).

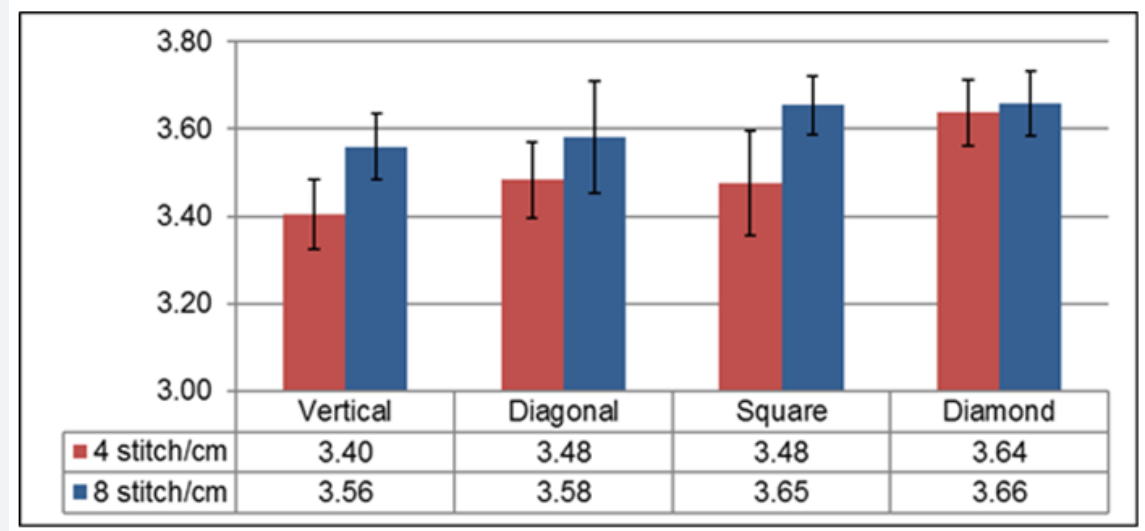

Figure 6: Puncture Load for 4-Layer (kN).

\section{Comparison on stitch density by ranking}

Comparison on stitch density by ranking was conducted to analyse which types of stitching pattern that presented dominant higher puncture load value.

From Table 1, the comparison on stitch density by ranking on the puncture load value was observed. Vertical stitched pattern has the higher puncture load for 4 stitches/cm samples and diagonal stitched pattern has the higher puncture load for 8 stitches/cm samples on 2-layer fabric.

Table 1: Puncture Load Ranking for 2-Layer (kN).

\begin{tabular}{|c|c|c|}
\hline Stitch Density & 4 stitches $/ \mathrm{cm}$ & 8 stitches $/ \mathrm{cm}$ \\
\hline Vertical & 1.71 & 2.09 \\
\hline Diagonal & 1.39 & 2.10 \\
\hline Square & 1.55 & 1.78 \\
\hline Diamond & 1.52 & 1.80 \\
\hline
\end{tabular}

From Table 2, it was found that diamond stitched pattern has the higher puncture load for 4 stitches/cm and diagonal stitched pattern has the higher puncture load for 8 stitches/cm on 3-layer fabric.
Table 2: Puncture Load Ranking for 3-Layer (kN).

\begin{tabular}{|c|c|c|}
\hline Stitch Density & 4 stitches/cm & 8 stitches/cm \\
\hline Vertical & 2.63 & 2.75 \\
\hline Diagonal & 2.54 & 2.85 \\
\hline Square & 2.52 & 2.51 \\
\hline Diamond & 2.64 & 2.50 \\
\hline
\end{tabular}

From Table 3, the diamond stitched pattern has the higher puncture load for both stitch densities on 4-layer fabric. From the analysis by ranking, it was observed that diamond and diagonal stitching patterns contributed much on the higher puncture load value for 4 stitches/cm and 8stitches/cm. Both types of stitching patterns are more flexible and stretchable since the condition of stitching in bias direction (45o angle) which makes the penetration load increased and allowed the fabric to withstand higher load of penetration. This indicated that the structure and configuration of the stitching patterns also gave significant effect to determine the suitability of the puncture resistance properties as a fabric system. 
Table 3: Puncture Load Ranking for 4-Layer (kN).

\begin{tabular}{|c|c|c|}
\hline Stitch Density & 4 stitches/cm & 8 stitches/cm \\
\hline Vertical & 3.40 & 3.56 \\
\hline Diagonal & 3.48 & 3.58 \\
\hline Square & 3.48 & 3.65 \\
\hline Diamond & 3.64 & 3.66 \\
\hline
\end{tabular}

\section{Fabric failure mechanism}

Figure 7-10 showed the surface morphology of the Twaron fabric after penetration of the puncture probe with the magnification of $30 x, 60 x$ and $100 x$. It can be observed that the structures of the warp and weft yarns were stretched, broken yarns occurred at the penetration area and the yarns were stretched near the penetration area. Figure 9-10 show the effect of penetration with the presence of the stitching yarns where warp and weft yarns were stretched and pressed towards the stitching area. The hole that was created from the tip of the puncture probe appeared on the stitching point. Broken yarns also occurred at the stitching area.

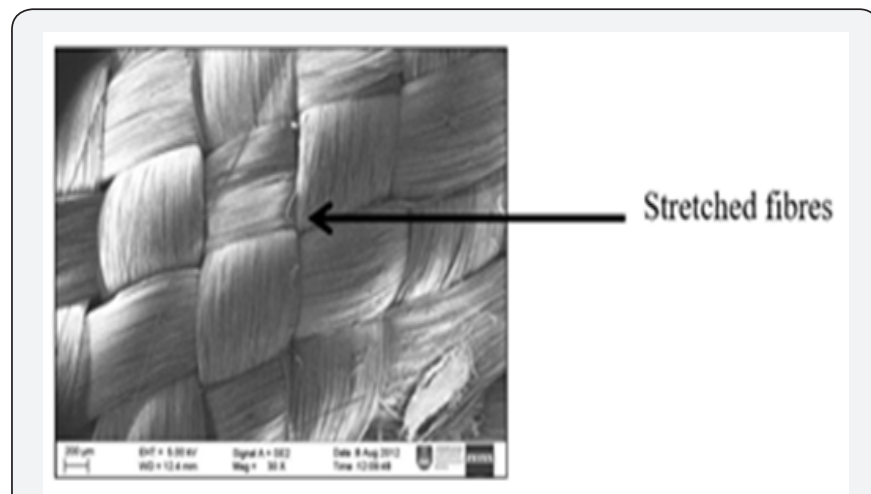

Figure 7: Surface Morphology at 30x Magnification.

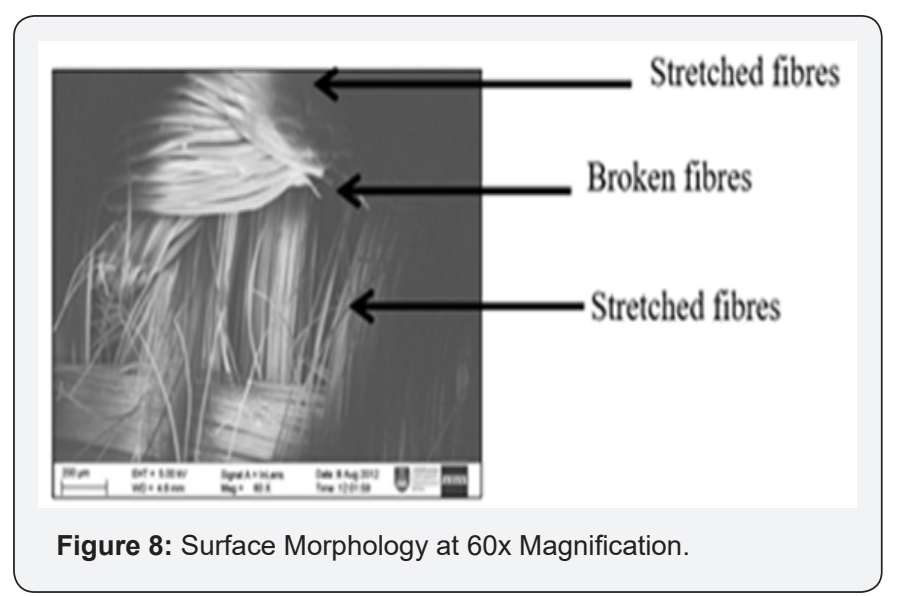

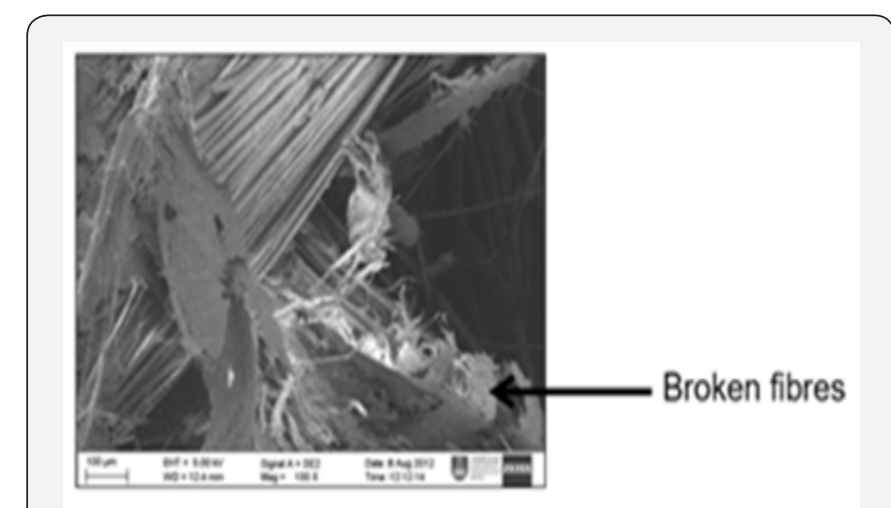

Figure 9: Surface Morphology at 100x Magnification.

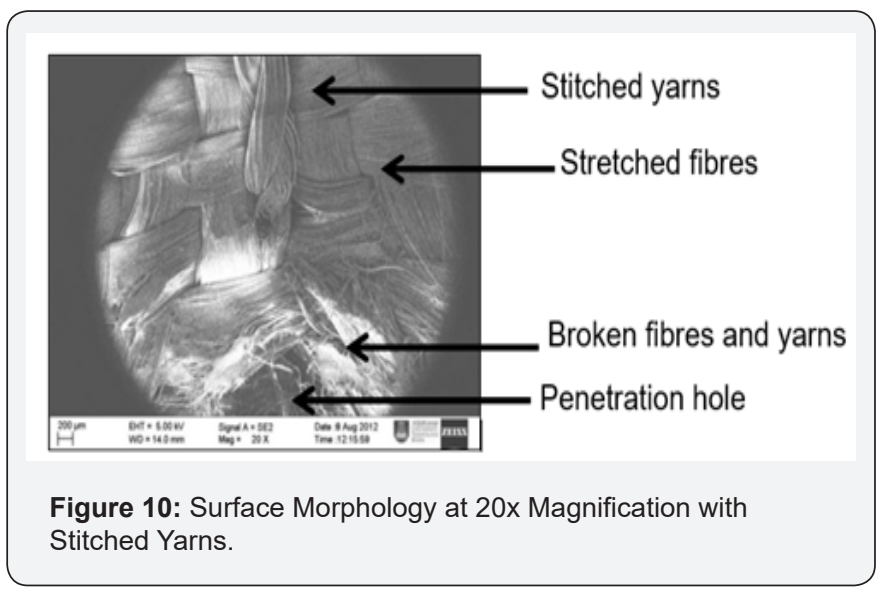

Figure 11-14 showed the close-up views on four types of stitching patterns after the penetration. The penetration hole showed localized damaged region, where the damage of the fabric restricted around the penetration area as the stitching yarn reduced the structural deformation and damage of the fabrics. The sharp probe tip penetrated the fabrics and pushing apart the fabrics to cut and break the fibres. The penetration shape is similar for all stitched fabric which exhibit circular shape of penetration.

There were three particularly different fabric failure modes in the slow penetration test which is local yam breakages, remote yarn failure, and yam pull-out. It was clear that the local yarn breakage was the main fabric failure mode in this study. Local yarn breakage is the breakage of all fibres at the point of penetration. It was mostly observed in the fabric failure mode, and followed by a sharp burst of sound and a sudden drop in the measured load. More than one yam failed and broke within a short time. It can be assumed that local yam breakage was the primary fabric failure mode for sharp-ended puncture probe. 


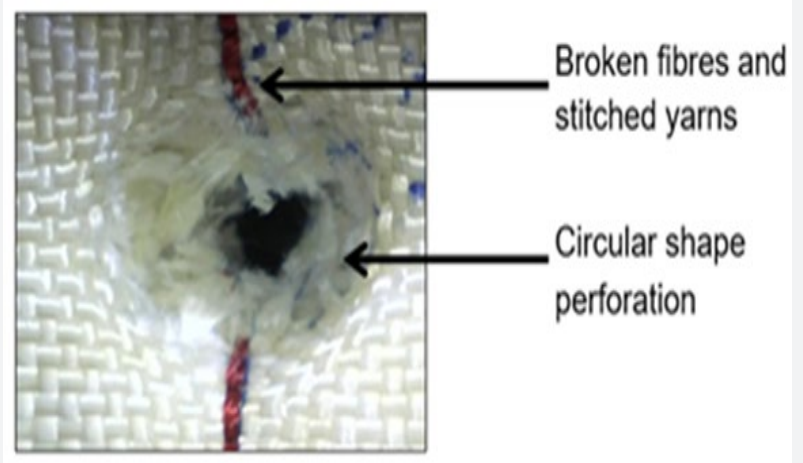

Figure 11: Close-up View of Vertical Stitched Pattern.

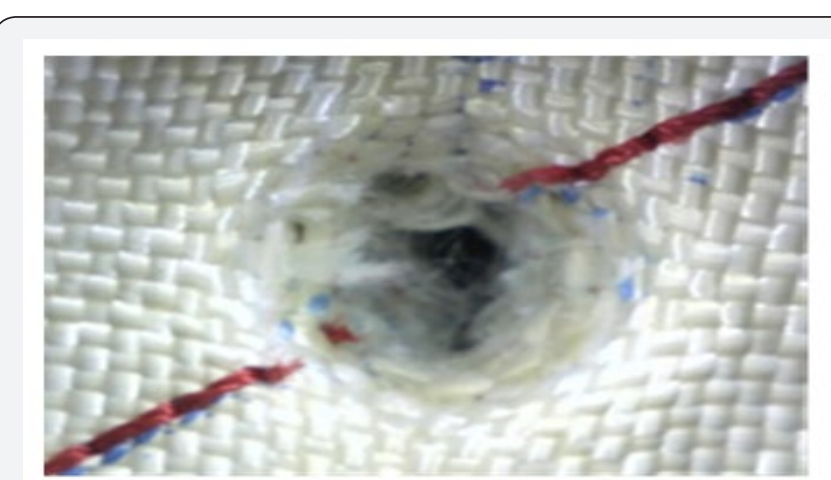

Figure 12: Close-up View of Diagonal Stitched Pattern.

\section{Conclusion}

The study found that fabrics that were stitched with high stitch density (8 stitches/cm) gave higher puncture load value from 2-layer to 4-layer fabric. The increment of fabric layer with higher stitch density could hold the multi-layer fabric better compared with the fabric that was stitched with lower stitch density. Furthermore, diamond and diagonal stitched patterns contributed much on higher puncture load value for 4 stitches/ $\mathrm{cm}$ and 8 stitches $/ \mathrm{cm}$. The structure of the stitching patterns as well as higher stitch density determines the suitability of the puncture resistance properties of a multi-layer fabric.

\section{Acknowledgements}

The authors would like to express great appreciation to the Institute of Research Management \& Innovation (IRMI) of Universiti Teknologi MARA (UiTM) for the Research Excellence Fund.

\section{References}

1. Tan KT, Yoshimura A, Watanabe N, Iwahori Y, Ishikawa T (2013) Effect of stitch density and stitch thread thickness on damage progression and failure characteristics of stitched composites under out-of-plane loading. Composites Science and Technology 74: 194-204.

2. Yudhanto A, Watanabe N, Iwahori Y, Hoshi H (2013) Effect of stitch density on tensile properties and damage mechanisms of stitched

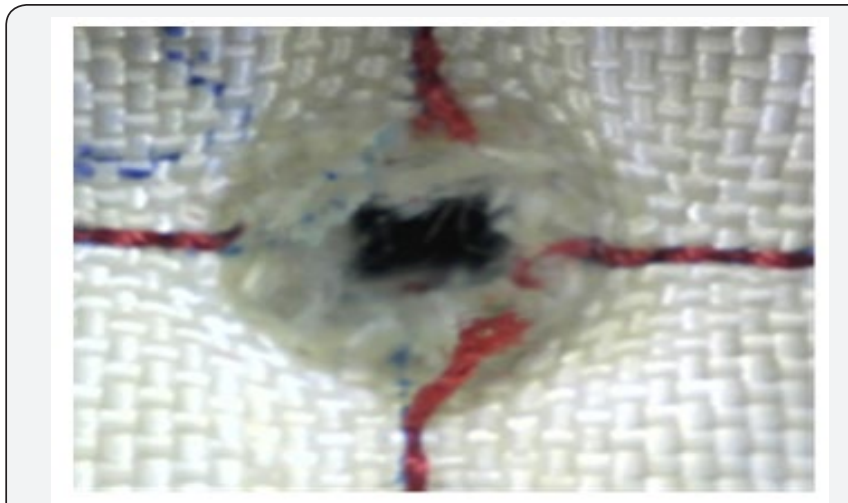

Figure 13: Close-up View of Square Stitched Pattern.

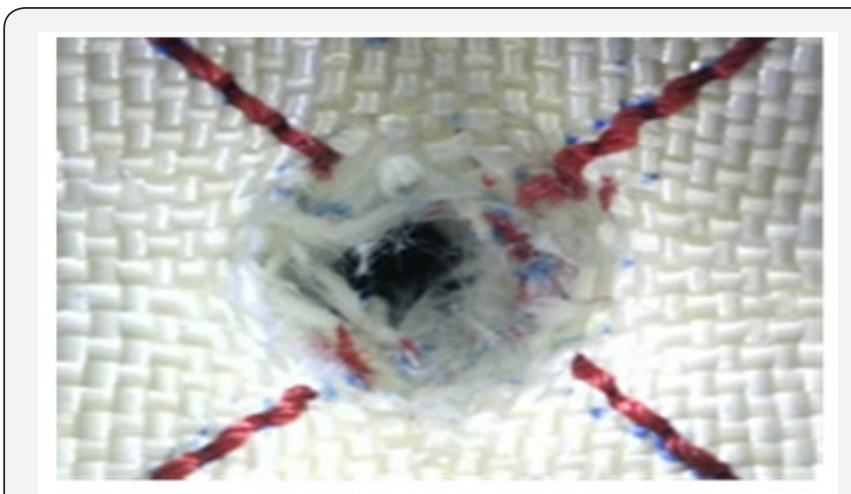

Figure 14: Close-up View of Diamond Stitched Pattern.

carbon/epoxy composites. Composites Part B: Engineering 46: 151165.

3. Karahan M, Ulcay Y, Eren R, Karahan N, Kaynak G (2009) Investigation into the Tensile Properties of Stitched and Unstitched Woven Aramid/ Vinyl Ester Composites. Textile Research Journal 80(10): 880-891.

4. Ahmad MR, Ahmad WYW, Salleh J, Samsuri A (2008) Effect of fabric stitching on ballistic impact resistance of natural rubber coated fabric systems. Materials \& Design 29(7): 1353-1358.

5. Nanthagopal V, Senthilram T, Dev VRG (2013) Flexural and impact studies on stitched self-reinforced polypropylene composites. Journal of Thermoplastic Composite Materials 27(11): 1504-1514.

6. Suhaimi SA, Salleh J, Ahmad MR, Ahmad NA, Bakar NAA (2012) Puncture resistance of Twaron fabric layers with different stitching patterns. International Journal of Textile Science 1(5): 44-48.

7. Carr DJ, Lankester C, Peare A, Fabri N, Gridley N (2012) Does quilting improve the fragment protective performance of body armour? Textile Research Journal 82(9): 883-888.

8. Karahan M, Ulcay Y, Karahan N, KuS A (2013) Influence of Stitching Parameters on Tensile Strength of Aramid/Vinyl Ester Composites. Materials Science 19(1).

9. Wang QS, Sun RJ, Tian X, Yao M, Feng Y (2016) Quasi-static puncture resistance behaviors of high-strength polyester fabric for soft body armor. Results in Physics 6: 554-560. 
Your next submission with Juniper Publishers will reach you the below assets

- Quality Editorial service

- Swift Peer Review

- Reprints availability

- E-prints Service

- Manuscript Podcast for convenient understanding

- Global attainment for your research

- Manuscript accessibility in different formats

( Pdf, E-pub, Full Text, Audio)

- Unceasing customer service

Track the below URL for one-step submission https://juniperpublishers.com/online-submission.php 